\title{
Design of a Small, Multi-Purpose, Autonomous Surface Vessel
}

\author{
Alexander Leonessa, Jeremiah Mandello, Yannick Morel, Miguel Vidal \\ Florida Atlantic University - Department of Ocean Engineering - Dania Beach, FL 33004-3023 \\ \{aleo, jmandell, ymorel, mvidal\}@seatech.fau.edı
}

\begin{abstract}
The continued development of Unmanned Underwater Vehicles (UUVs) has also brought about new complex missions that the vehicles must perform. Such missions include mine counter measures (MCM), underwater system inspection, route surveying, and oceanographic sampling. More recently, a growing need for missions to be performed by multiple vehicles has emerged. An important task in being able to perform such a multi-vehicle operation is for each vehicle's position and orientation to be precisely known and updated to onboard sensors, in real-time, maximizing the surreptitious capabilities and quality of the data. Without this high quality navigation data, the mission performance is poor and little confidence can be given to the results.
\end{abstract}

Currently, UUVs can use inertial navigation systems, coupled with Doppler Velocity Loggers (DVLs), for navigation. However, inertial navigation systems are large, high-power, and expensive, while DVLs have limited bottom-tracking range. Some UUVs are equipped with global positioning systems (GPS), however the vehicle must surface to establish either a RF or satellite communication link. A solution, to increase the accuracy of a vehicles position is the use of a surface vessel, notably an autonomous surface vessel (ASV). One such vehicle is currently under development by the Department of Ocean Engineering at Florida Atlantic University. The ASV is being built upon an existing surface vessel navigation and control package, a vertical communication and US BL navigation system derived from the FAU-Dual Purpose Acoustic Modem and 3D motion compensation algorithm that utilizes a low cost GPS/IMU/COMPASS/ADCP system. The accurate positioning system onboard the ASV uses an acoustic uplink system between the ASV and UUVs below, to provide the UUVs with the navigation information needed for a successful mission.

The development of the ASV presents several problems such as controlling the vehicle, so that it can operate autonomously, gathering the sensor information, and passing the information to the UUVs below, in real-time. The control of the ASV can be divided into software and hardware components. On the software side, Simulink, part of Matlab, allows a user to develop a controller for the ASV by using a friendly graphical user interface (GUI). Matlab also allows for a host to target communication, either through RS -232 or TCP/IP, by using a toolbox called xPC Target. This setup allows the controller to be developed and compiled on a personal computer, the host, and then downloaded to a PC-104 stack inside the ASV, the target. Simulink blocks can also be created to control the flow of information from the sensors to the PC104 stack, whether the sensors are connected via an AD/DA board or through serial ports using RS232 communication. While the ASV is designed to give UUVs a more accurate position without installing expensive equipment on each of the UUVs, it can also be used so that a user can monitor the progress of a mission. The communication from the user to the ASV is also accomplished using XPC Target's, host to target communication using a wireless link, and from the ASV to the
UUVs using an acoustic modem. By using a surface vehicle, the mission performance of a group of UUVs can be improved by providing accurate and up-to-date positioning as well as by allowing a user to change the mission on the fly, without having to recover the UUVs.

\section{INTRODUCTION}

A limitation in the use of unmanned underwater vehicles (UUV) is their inability to receive accurate position measurements through global positioning satellites while underwater. This leads to inaccuracies when performing standard missions, such as; mine counter measures (MCM), underwater system inspection, route surveying, and oceanographic sampling. In helping to counter these limitations, sensors such as Inertia Measurement Units (IMUs) and Doppler Velocity Loggers (DVLs) are used to estimate the position of the UUV however these sensors alone cannot solve the problem. While the IMU can give accurate data for a vehicles' acceleration, upon integrating this data errors will appear in the derived velocity and position vectors. Using data from the DVL will help to eliminate some of this error, but not all. The DVL is also limited in its bottom-tracking range. To better improve the ability for multiple UUVs to perform tasks efficiently, each vehicle needs to know its precise location. To accomplish the task of relaying accurate position values to a non-stationary underwater vehicle the design of an autonomous surface vessel (ASV) was begun. The ASV does not limit the range or area that a UUV can operate in as this vehicle is a mobile platform that can follow the UUVs during their mission. In the development of a vehicle to accomplish this task the design processes can be separate into three parts, mechanical, electrical/hardware and software. The mechanical design of the vehicle includes the size of the vehicle, hull form and housing for the electronic components and batteries. The electrical design includes all sensors, computers, motors and batteries needed to accomplish the task at hand. The software portion of the paper does not deal with the development of a controller for the vessel, but instead focuses on using XPC Target, a software package from MathWorks, used for data acquisition in conjunction with vehicle control.

\section{MECHANICAL DESIGN}

\section{A. Preliminary Design}

The three major design constraints for this vehicle where determined to be the vehicle's stability, size, and the ability to operate for twenty-four hours at a cruise speed of five knots. A trade study was used to evaluated dfferent hull forms based on sensor motion, volume, cost, controllability, ease of 
manufacture, launch and recovery, complexity, portability, stability, draft, drag, speed and weight. From the five hull forms (catamaran, trimaran, streamlined mono-hull, troller mono-hull, and semi-submersible) that were considered, the catamaran proved the best, with the semi -submersible a close second.

Upon deciding on the catamaran hull, a small waterplane area twin hull (SWATH) design and a catamaran with a deep keel were studied. The SWATH design was ruled out due to concern over the vehicle's stability when encountering waves, because of the small size of the ASV. A small catamaran with a deep keel was found to best meet the design requirements for a small autonomous surface vessel. This design allows the instrument payload, batteries and computer to be housed below the surface. A Pro-E drawing of this can be seen in Fig. 1 .

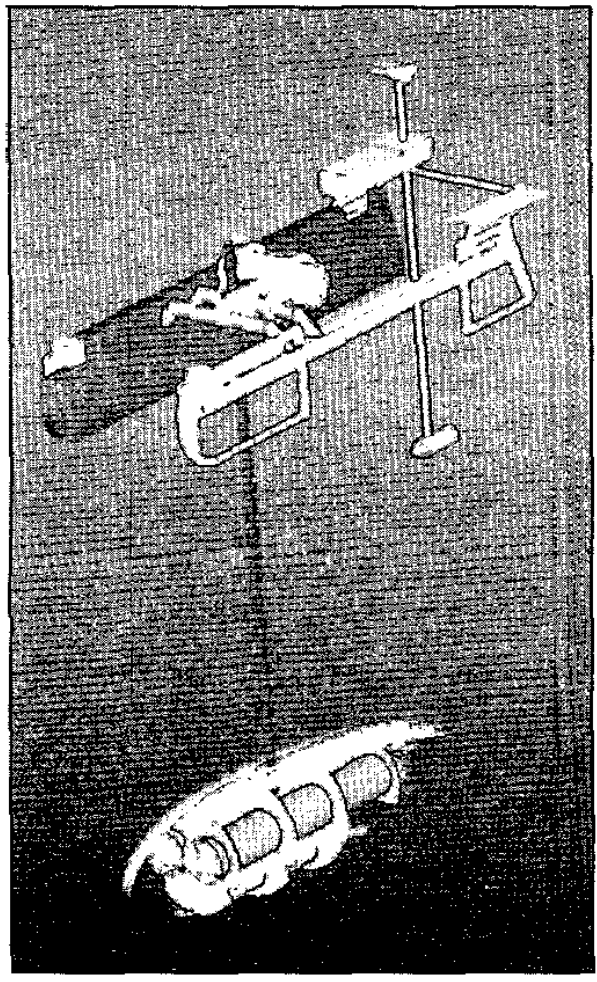

Fig. 1. Autonomous Surface Vessel Model

\section{B. Parametric Modeling}

Once the hull form was selected, a more in-depth analysis needed to be performed to determine the exact size of the vehicle. Since there were several variables that were dependent on each other, a parametric model was developed using Matlab. The variables studied were resistance, battery volume, instrument payload size, surface pontoons size, and the hydrostatics of the instrument payload and surface pontoons.

The total resistance for a ship is comprised of frictional, wave, eddy and air resistance. When finding the total resistance for the ASV, wave and eddy resistance were combined as residual resistance, and air resistance was neglected. The resistance was then found for the three main components of the vessel; surface pontoons, instrument payload, and the connecting strut.

The frictional resistance, $R_{f}$, of the surface pontoons was estimated, considering the surface pontoons as thin plates, using

$$
R_{f}=\frac{1}{2} \rho U^{2} S C_{f} C_{f o r m}
$$

Where? is the water density, $U$ is the vehicle's velocity, $S$ is the area, $C_{f}$ is the coefficient of frictional resistance, and $C_{f o r m}$ is the coefficient of hull efficiency which needs to be included since the surface pontoons are not plates. These variables hold true for all of the following equations unless otherwise noted. The residual friction, $R_{r}$, of the surface pontoons was estimated considering the pontoons to be a Wigley hull.

$$
R_{r}=\frac{1}{2} \rho U^{2} S C_{w} .
$$

In (1.2) $C_{W}$ is defined as the wave resistance coefficient. When determining the frictional and residual friction for the instrument payload and connecting strut the equations are of the same form as (1.1) and (1.2).

The total resistance for the ASV is the sum of residual and frictional resistance for all three sections. The total resistance for each component and for the vehicle can be seen in Fig. 2.

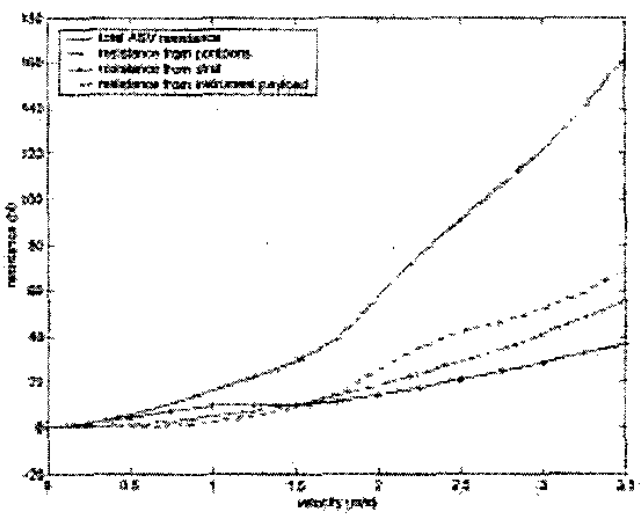

Fig. 2. Resistance estimate for the ASV

\section{Structural Design}

Upon determining the total resistance for the ASV, for a given size, the needed battery volume was determined. The power needed for the vessel to operate for twenty-four hours at five knots was estimated based on the vessel's resistance. Using the estimated needed power requirements and the volumetric efficiency of the battery chemistry, the corresponding battery volume was estimated. This battery volume was used to determine the size and weight of the instrument payload. The size of the surface pontoons was then estimated based on the wet weight of the instrument payload. The size of the surface pontoons and instrument 
payload was finally used to estimate the resistance of the vehicle. The parametric model took these factors into account, optimizing the vehicle to meet the size and length of operation constraints.

With the hull form and size of the vehicle now determined, the structural design of the vehicle had to be performed. The structural design of the vehicle could again be broken into the same three parts; surface pontoons, instrument payload, and connecting strut.

The surface pontoons need to generate a large amount of displacement to counter the overall weight of the vehicle. Generally hulls are built with a structural shell that displaces water, generating the needed displacement. The shell is reinforced with stringers and bulkheads where loads are expected. While this method works well, it can be a time consuming manufacturing process. To avoid this time consuming process an alternative design was proposed and ultimately chosen. This alternative design called for a frame, to provide structural integrity, cast in foam, to provide floatation. This can be seen in Fig. 1. To manufacture the surface pontoons, an aluminum frame was assembled. A female mold was then cut in the shape of the pontoons and placed around the frame. Liquid foam was then poured into the mold and allowed to harden, a process that took less than ten minutes. The mold was then removed leaving the foam pontoons permanently attached to the aluminum frame.

Before the manufacturing process could take place the frame had to be designed and analyzed. The frame was designed to not only connect the two pontoons, but also to provide support for the foam. After determining a general layout of the frame, ANSYS was used to determine the needed wall thickness and placement of gussets for extra strength. A screenshot of the analysis using ANSYS can be seen in Fig. 3.

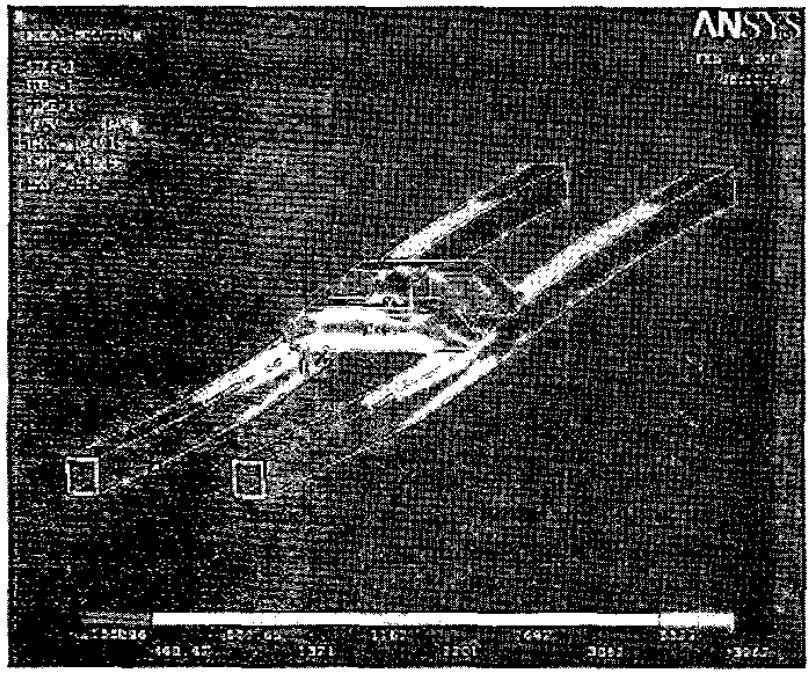

Fig. 3. Frame analysis using ANSYS

The frame itself is a combination of sheet, box and tube aluminum. The two pontoons each have a box aluminum stringer to prevent the foam from snapping at its weakest point. Since the stringer would be in the upper portion of the pontoon an aluminum sheet was machined and welded to the stringer as a perimeter support for the foam. A mounting plate for motors was welded to each side of the frame at the aft end. The frame connects both pontoons using schedule 80 tubing to resist torsion.

The instrument payload was designed to allow for several pressure vessels to be covered by a streamlined shell, which is not water-tight. In designing the instrument payload it was important to develop a structure that did not allow any of the instruments to shift. To establish a solid base for the instrument payload a three-eighths inch plate of aluminum was used as the backbone. Four disks of three-quarters inch high-density polyethylene where hung. The pieces of polyethylene were machined so as to hold the pressure vessels. The sections of polyethylene were also joined together by half-inch aluminum rods, increasing the polar moment of inertia of the support structure. ANSYS was used to study several variations of the frame including an all aluminum version. The chosen design was, for all practical purposes, as strong as the other proposed designs, but had the advantages of a lower dry weight and cost.

The connecting strut was designed to be strong enough to handle the mass and inertia of the instrument payload, hollow so that wires can pass from the instrument payload to the surface pontoons, and to have as little resistance as possible. Several possible materials were studied and chrome-moly was chosen for its superb strength. It is important to note that this material had to be powder-coated to protect it from oxidation. The strut was designed to have a minor axis of 1inch, a major axis of 2.36-inches, and a wall thickness of 0.049 -inches. The cross section of this srut can be seen below in Fig. 4.

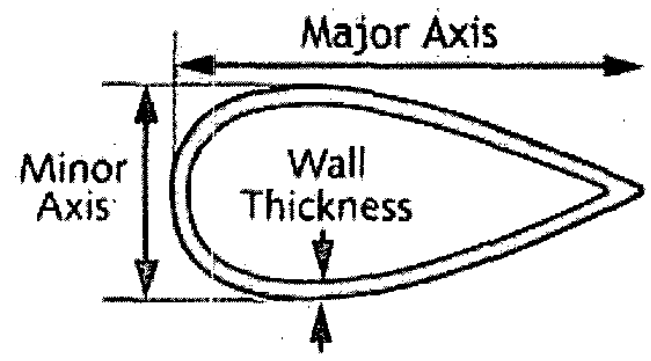

Fig. 4. Cross section of connecting strut.

\section{ELECTRONICS}

A. Instruments

The main purpose of the sensors is to provide relevant and sufficient data allowing for an accurate estimate of the vehicle's state (position, attitude, velocity, and angular velocity). The sensors act as an interface between the ASV and the environment, providing the vehicle with information relative to the environment. The first step in using the ASV to provide accurate position information to UUVs is for the ASV to accurately know its own position and orientation. To accomplish this goal the ASV has been equipped with the following instruments; Acoustic Doppler Current Profiler (ADCP), electronic compass, Differential Global Positioning 
System receiver (DGPS), Inertial Measurement Unit (IMU), a Dual Purpose Acoustic Modem (DPAM), and a wireless Ethernet connection.

The ADCP is used to measure the vehicle's body-fixed longitudinal, lateral, and vertical velocities with respect to the sea bed. It can also be used to provide measurements of the vehicle's attitude, altitude, earth-fixed longitudinal, lateral and vertical velocities, as well as the temperature of the sea water. As mentioned previously a limitation on this device is its bottom-tracking range. If the depth to the sea floor exceeds this range then the device only determines the velocity of the vehicle relative to the surrounding water. The ADCP in use on the ASV is a Workhorse Sentinel from RD Instruments and it communicates with the onboard computer through serial communication (RS232).

The electronic compass measures the vehicle's attitude in terms of heading, pitch and roll. The information provided by the compass is obviously redundant with the attitude measurements provided by the ADCP. However this redundancy allows for a refining of the vehicle's state. The electronic compass in use is a TCM2 from Precision Navigation and it communicates using RS232.

The DGPS is used to provide an estimate of the vehicle's position in terms of latitude and longitude, using the GPS - satellite constellation. Unlike the other devices, the DGPS must be housed on the surface pontoons and not in the instrument payload. The DGPS in use is a DGPS MAX from CSI Wireless which communicates through RS232.

The IMU measures the vehicle's longitudinal, lateral, and vertical acceleration, as well as its angular velocity in heading, pitch and roll. Unlike the previously mentioned instruments this device communicates through analog signals. These signals are filtered by a bank of DP68 Low-Pass Filters from Frequency Devices. The filtered signals are then fed into an analog to digital converter. The IMU in use is a MotionPak from BEI Electronics.

The ASV communicates with UUVs through an acoustic system. This acoustic system consists of a pair of transducers used for transmission and reception, an array of transducers in an Ultra Short Base Line (USBL) configuration, and a Dual Purpose Acoustic Modem (DPAM), which was developed at Florida Atlantic University. The DPAM uses a transmit transducer and a receive hydrophone to communicate with an UUV, while the USBL array is used to determine the relative position of the UUV to the ASV. The DPAM communicates with the computer onboard the ASV through RS232 serial communication.

Another device used on the ASV is a BreezeNet Pro.11 Series wireless Transmission Control Protocol / Internet Protocol (TCP/IP) system, from Alvarion. This device was selected because the computer onboard the ASV is the target in a host/target framework, which will be further explained later, where communication between the host and target is important. Although the target, in this framework can run independently, it is still desired for the host to have a certain degree of control over the target. Indeed the host can be used to start or stop the mission, reboot the target, monitor the application running on the target and tune this application on the fly. All of these functionalities are a necessity during the testing process and can still be useful in future applications. The BreezeNet wireless system is composed of a pair of transmitters / receivers, or access points, one of which is connected to the host computer and the other to the target. Since the ASV is the target the BreezeNet access point is mounted on the surface pontoons, so that the antennas are above the sea surface.

\section{B. Actuators}

The propulsion system of the ASV consists of a pair of off the shelf trolling motors. These, Riptide trolling motors, from Minn Kota, use up to 900 watts of power. However providing them with about ten percent of that amount will allow the vehicle to reach its cruise speed. The two motors, mounted on the pontoons, allow the vehicle to be steered by setting a different RPM for each motor. The steering is further facilitated by the fact that each motor can rotate 360 degrees. In the retail state of these motors, the RPM and motor orientation are controlled by a foot pedal which had to be modified. The motor is now controlled by a High Performance Standard Node (HPSN). A HPSN is classically used as a node in a Lonwork network, but its capacity to generate digital and analog signals was utilized to control the motors. A further modification was made in the addition of a compass on each motor, allowing the relative orientation of the motor to be measured with respect to the vehicle. These compasses are Vector Electronic Modules from Precision Navigation.

\section{Computer}

The selection of the onboard computer was strongly influenced by the embedded nature of the system. The onboard electronics had to be compact enough and feature reasonably low power consumption. For these reasons, the onboard computer was chosen to be in the $\mathrm{PC} 104$ format, one of the most commonly used standards for embedded systems. Not only does this format satisfy the aforementioned criteria, but the fact that it is widely used makes it easy to find any type of board, featuring any functionality one might need. The ASV's onboard computer consists of several boards; a MOPS1cd6 computer, an Emerald-MM serial hub, a Diamond-MM-32-AT Analog to Digital and Digital to Analog (AD/DA) converter, and a HE104 Drect Current to Direct Current (DC/DC) converter.

The MOPS1cd6, from JUMPtec, has a CPU clock speed of $166 \mathrm{MHz}$. It also features two serial ports, a parallel port, Ethernet access, and supports both a keyboard and a VGA screen. The device also has 32 megabytes of flash disk memory, from SunDisk.

Since most of the instrumentations communicate to the computer through RS232 serial communication, a serial hub was needed. The chosen serial hub is an Emerald-MM, from Diamond Systems. This device has four serial ports, which increases the number of serial ports on the computer to six.

As previously mentioned, the IMU outputs analog signals, so an $\mathrm{AD} / \mathrm{DA}$ converter is needed, allowing the IMU to send data to the computer. The Diamond-MM-32-AT can be configured to have 16 differential channels, 32 singleended channels, or 8 differential and 16 single-ended 


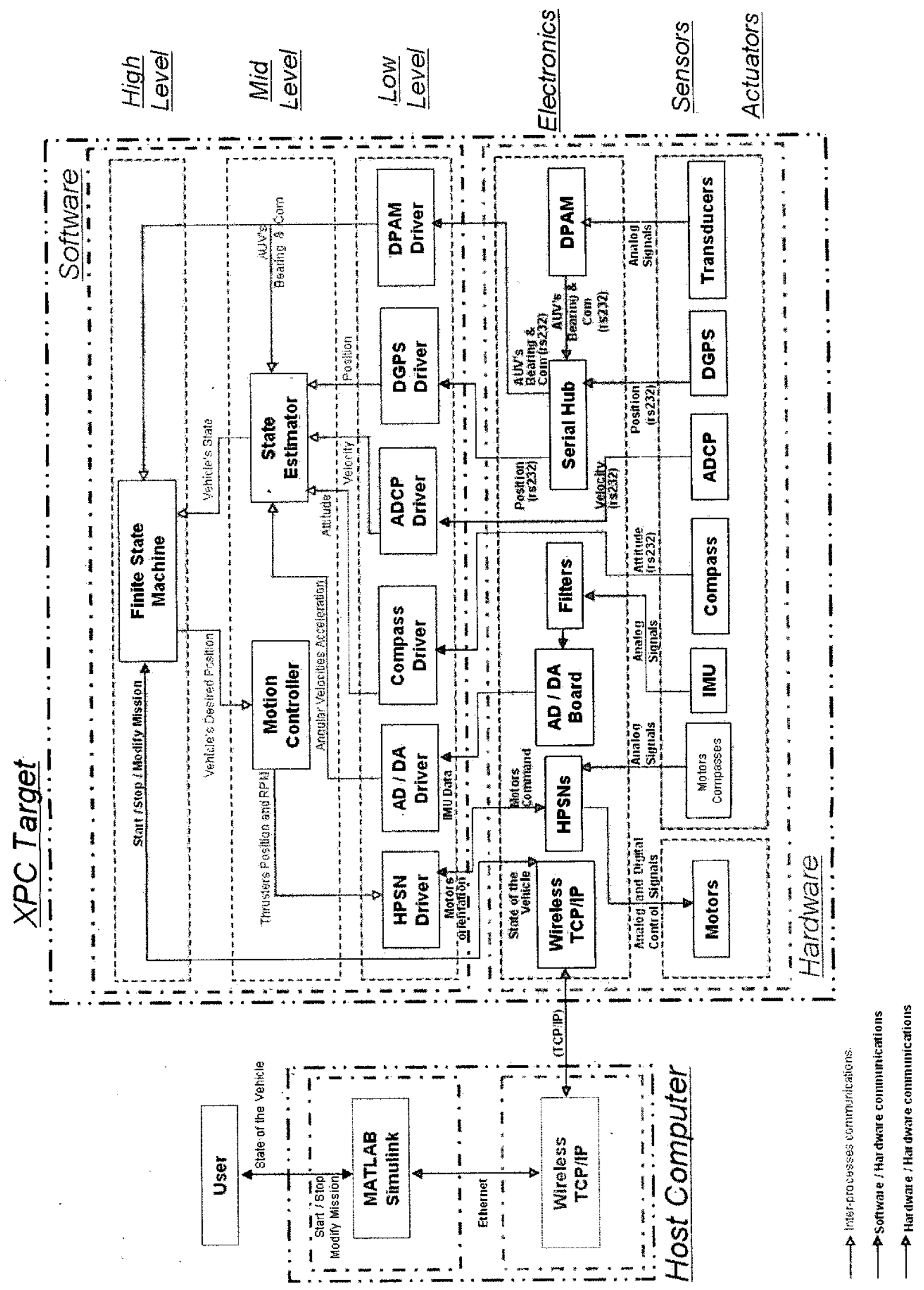

Fig. 5. ASV's signals block diagram. 


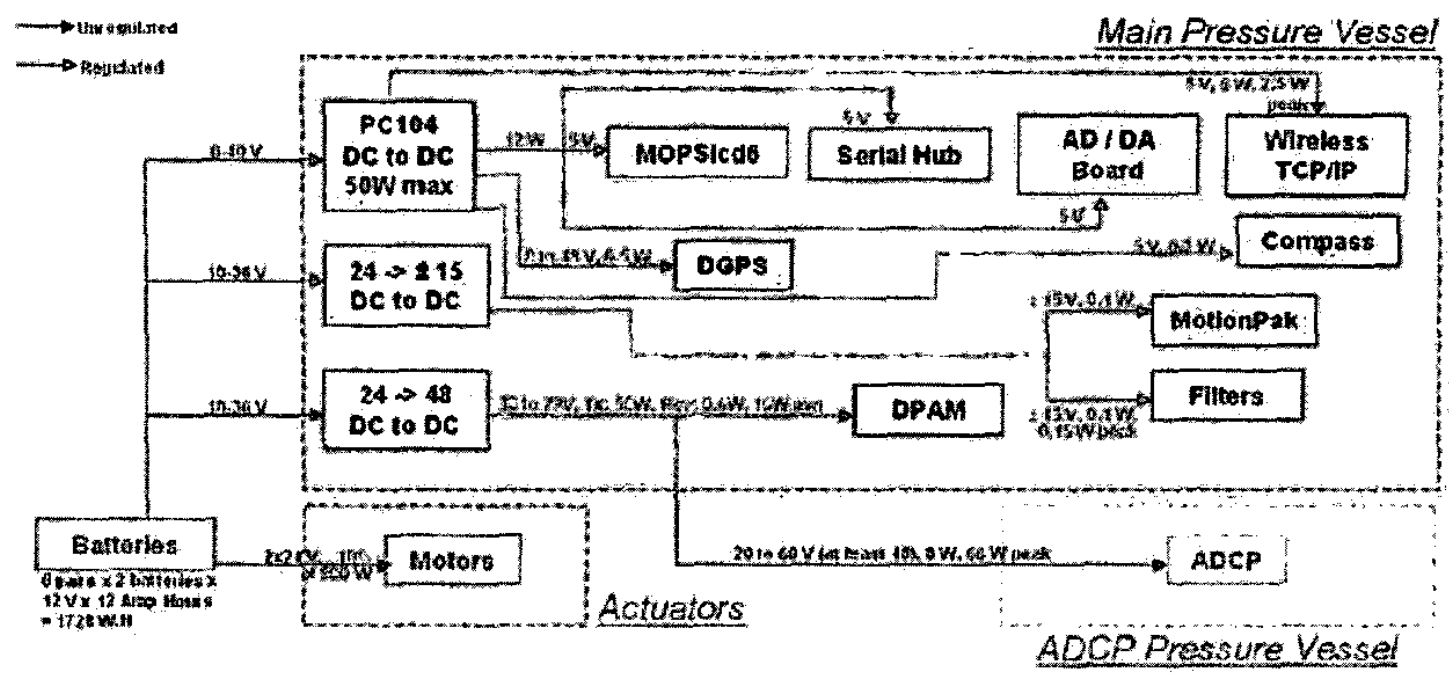

Fig. 6. Power distribution block diagram

channels. This flexibility was desirable since it would allow for easier future expansions.

To ensure that the onboard computer is supplied with a stabilized five volts a HE104 high efficiency DC to DC converter, from Tri-M Engineering, is used. This device uses an input voltage between 6 and 40 volts (V), and outputs stabilized $5 \mathrm{~V}, 12 \mathrm{~V},-5 \mathrm{~V}$ and $-12 \mathrm{~V}$. This allows the device to supply power not only to the computer, but to several instruments as well. The interaction between all of the instruments the onboard computer and the host and target can be seen in Fig. 5 .

\section{Power}

It was determined, based on length of operation time, that six pairs of twelve volt batteries (the used output voltage being $24 \mathrm{~V}$ ) would be needed. The batteries that were selected are NP12-12 from Yuasa and they are lead acid based. Since each battery provides 12 Amp-hours (Ah), the implied total Watt-hours (Wh) for the ASV is 1728 Wh.

These batteries are housed in two pressure vessels, which are located in the instrument payload.

With the batteries and the computer's DC/DC converter, the ASV can supply instruments with $24 \mathrm{~V}$ unregulated, $5 \mathrm{~V}$, $12 \mathrm{~V},-5 \mathrm{~V}$, and $-12 \mathrm{~V}$ regulated. However several instruments required specific voltages that were not available from the batteries or the DC/DC converter. In particular the IMU needs $\pm 15 \mathrm{~V}$ and both the DPAM and the ADCP require an input voltage higher than $24 \mathrm{~V}$. Two extra DC/DC converters had to be integrated into the system because of this. The $\pm 15 \mathrm{~V}$ regulated is supplied by a BWR A Series DC/DC converter, from Datel. This device accepts an input voltage from 18 to 36 volts, has a typical efficiency of $84 \%$, and can output up to 15 watts. The power for the DPAM and ADCP is provided by a DC/DC Converter Module from Vicor. This device uses an input voltage that can range from 18 to 36 volts, has a typical efficiency of $87 \%$, can output 100 watts and has a regulated output of 48 volts. These two DC/DC converters were chosen not only for their high efficiency but also for their compact packaging. 'The Datel converter is 1 inch by 2 inches by 0.48 inches and the Vicor converter is 2.28 inches by 1.45 inches by 0.5 inches.

The power distribution on the presented vehicle is simple. The batteries supply unregulated 24 volts. This is unregulated voltage is used on one side by the two motors and on the other side s converted to the various voltages utilized by the sensors and electronic systems. This has been illustrated in Fig. 6.

\section{SOFTWARE: XPC TARGET}

\section{A. General Information}

A recent addition to MathWorks', Matlab software is xPC Target. This toolbox is designed to allow a user to run code generated from Simulink, another Matlab toolbox. Simulink can be used to create simulations, develop control systems and/or for data acquisition. Simulink is based on a Graphical User Interface (GUI) that allows for the easy development and debugging of control and data acquisition systems. As mentioned previously, xPC Target is based on a host/target configuration, where the host computer has the full version of Matlab and the target computer utilizes a kernel created by the host machine. The kernel allows the target to connect to the host machine, download code created on the host machine and then run that code. This allows for the elimination of a traditional Operating System (OS) on the ASV.

\section{B. Host / Target Communication}

As previously mentioned the host / target communication is an important component when using $x P C$ Target. Although the target can run independent of the host once the code has been downloaded to it, the presence of a communications link allows features, such as target monitoring, data logging to the host's hard-drive, and the ability to change certain parameters within the code. The 
communications link between the host -and target can be accomplished using either RS232 or TCP/IP protocols.

\section{Hardware Support}

When using xPC Target, a new element is added to hardware selection. While drivers have been provided with $\mathrm{xPC}$ Target for some hardware, there are many devices that are not currently supported. That is not to say that these devices cannot be used with this software. Simulink allows for the creation of $S$ functions which can be used to create drivers for unsupported hardware. The S-function block allows the user to define a Simulink block by linking code written in $\mathrm{C}, \mathrm{C}++$ or mfile to that block. It is important to note that an S-function linked to an mrfile cannot be used in conjunction with Real Time Workshop (RTW). When integrating the hardware in use on the ASV, drivers where provided in $\mathrm{xPC}$ Target for the Diamond-MM-32 AD/DA converter, however drivers where not provided for the Emerald-MM serial hub. An S-function was used to create the drivers for this device, which can be a very time consuming process. While xPC Target does not support any specific instruments per se, but instead uses drivers to control the lines of communication (serial ports or AD/DA channels). The driver written for the serial hub allows communication between the onboard computer and the ADCP, electronic compass, DGPS and acoustic system. While the supplied driver for the $\mathrm{AD} / \mathrm{DA}$ converter allows communication between the onboard computer and the IMU.

\section{Data logging}

Data logging allows the user to obtain information gathered by various sensors including information about the system's state under varying conditions. While it has been stated that the target can run independent of the host, xPC Target, in its current form (2.0), only supports data logging to the host's hard-drive. 'This would not prove to be a problem for stationary systems, but when dealing with the ASV it poses a problem. The addition of the BreezeNet wireless Ethernet system allows the ASV to communicate with the host computer, within a certain range, while maintaining the maneuverability needed by the vehicle. Recently another problem was discovered while logging data across the wireless Ethernet. The Simulink blocks provided in $\mathrm{xPC}$ Target does not allow the data to be logged in real time. The provided blocks are linked to code written as an $\mathrm{m}$-file, and as previously stated this is not compatible with Real Time Workshop. Several solutions to this problem are currently under investigation so that the ASV will have the ability to log data in real time.

\section{CONCLUSION}

The Autonomous Surface Vessel (ASV) has been designed to provide a platform allowing Unmanned Underwater Vehicles (UUVs) to better know their position. To accomplish this task the ASV had to accurately know its own position, and as such was designed to have all of the instruments needed to accomplish this goal. Next the ASV must be able to communicate with the UUVs allowing them to determine their position based on the position of the ASV. This task has been accomplished through the use of an acoustic system that is composed of the Dual Purpose Acoustic Modem (DPAM) and an array of transducers in an Ultra Short Base Line (USBL) configuration. This provides better underwater tracking for the UUVs, resulting in more accurate and efficient search patterns. Now a UUV in communication with the $\mathrm{ASV}^{\cdot}$ does not need to interrupt their mission by rising to the surface to receive their position via Global Positioning Satellites.

\section{ACKNOWLEDGEMENTS}

We wish to thank the Office of Naval Research for their support and interest in this project. 